\title{
EEG-fMRI of Idiopathic and Secondarily Generalized Epilepsies
}

Khalid Hamandi ${ }^{1}$, Afraim Salek-Haddadi ${ }^{1}$, Helmut Laufs ${ }^{1}$, Adam Liston ${ }^{1}$, Karl Friston ${ }^{2}$, David R Fish $^{1}$, John S Duncan ${ }^{1}$, Louis Lemieux ${ }^{1}$

Author affiliations:

1. National Society for Epilepsy and Department of Clinical and Experimental Epilepsy, Institute of Neurology, University College London, Queen Square, London, United Kingdom.

2. The Wellcome Department of Imaging Neuroscience, Institute of Neurology, Queen Square, London WC1N 3BG, United Kingdom.

Key Words: EEG-fMRI, idiopathic generalized epilepsy, thalamocortical system

Corresponding author:

Khalid Hamandi

MRI Unit, National Society for Epilepsy

Chalfont St Peter

Buckinghamshire

SL9 0RJ

Telephone: $\quad+441494601300 \quad$ Fax: $\quad+441494875666$

Email: k.hamandi@ion.ucl.ac.uk

Number of: references 68, figures 4, tables 3.

Word count: Title 7, Abstract 193, Main text 3208

Running title: EEG-fMRI of GSW 


\begin{abstract}
We used simultaneous EEG and functional MRI (EEG-fMRI) to study generalized spike wave activity (GSW) in idiopathic and secondary generalized epilepsy (SGE). Recent studies have demonstrated thalamic and cortical fMRI signal changes in association with GSW in idiopathic generalized epilepsy (IGE). We report on a large cohort of patients that included both IGE and SGE, and give a functional interpretation of our findings. Forty six patients with GSW were studied with EEG-fMRI; 30 with IGE and 16 with SGE. GSWrelated BOLD signal changes were seen in 25 of 36 individual patients who had GSW during EEG-fMRI. This was seen in thalamus (60\%) and symmetrically in frontal cortex (92\%), parietal cortex (76\%) and posterior cingulate cortex / precuneus (80\%). Thalamic BOLD changes were predominantly positive and cortical changes predominantly negative. Group analysis showed a negative BOLD response in the cortex in the IGE group and to a lesser extent a positive response in thalamus. Thalamic activation was consistent with its known role in GSW, and its detection with EEG-fMRI may in part be related to the frequency and duration of GSW epochs recorded. The spatial distribution of the cortical fMRI response to GSW in both IGE and SGE involved areas of association cortex that are most active during conscious rest. Reduction of activity in these regions during GSW is consistent with the clinical manifestation of absence seizures.
\end{abstract}




\section{Introduction}

Generalized spike wave (GSW) activity, is the electroencephalographic (EEG) hallmark of idiopathic generalized epilepsy (IGE), occurring in runs of 2.5-4 $\mathrm{Hz}$ spike and slow wave activity, typically arising from a normal background EEG \{Duncan, 1997\}. GSW is also seen in symptomatic generalized epilepsies where it is usually associated with an abnormal background EEG, and clinical evidence of other neurological dysfunction $\{$ Holmes et al., 1987\}.

The pathophysiological substrate of GSW remains enigmatic. The debate between a subcortical origin "the centrencephalic hypothesis" $\{$ Jasper and Drooglever-Fortuyn, 1947\} versus a cortical origin \{Marcus and Watson, 1968\} was reconciled to an extent by the corticoreticular hypothesis. This proposed a role for both cortex and subcortical structures \{Gloor, 1968\}, with aberrant oscillatory rhythms in reciprocally connected thalamocortical loops normally involved in the generation of sleep spindles \{ Gloor, 1968 \}, leading to GSW. The primary neuroanatomical and neurochemical abnormality in IGE remains undetermined with evidence and arguments for onset in either cortex \{Timofeev and Steriade, 2004\} or thalamus \{Avoli et al., 2001\}. Much of the evidence pertaining to the pathophysiology of GSW comes from invasive electrophysiological and neurochemical recordings in animals \{Avoli et al., 2001\}. A small number of intracranial studies have been reported in man in which spike wave activity was recorded in both thalamus and cortex \{Williams, 1953\} \{Niedermeyer et al., $1969\}$ \{Velasco et al., 1989\}. The spatial sampling of depth studies is limited to the immediate vicinity of the implanted electrodes, and their invasiveness, in the absence of clinical benefit precludes their current use in IGE. 
Combining EEG recording with fMRI (EEG-fMRI) enables the non-invasive mapping of haemodynamic correlates of specific EEG events or rhythms \{Salek-Haddadi et al., 2003a , by means of the blood oxygen level dependent (BOLD) contrast \{ Ogawa et al., $1990\}$. Studies in patients with focal epilepsy have demonstrated spatially concordant BOLD activations in relation to focal epileptiform discharges (IED), evidence that EEGfMRI can provide localizing information on generators of these discharges \{warach $1996\}\{$ Krakow et al., 1999\} \{seeck 2000\} \{Lemieux et al., 2001\} \{jager 2002\}\{Al Asmi et al., 2003\}. Whether EEG-fMRI is able to shed further light on the pathophysiology of GSW, by localizing generators of these discharges remains to be seen.

Salek Haddadi et al \{Salek-Haddadi et al., 2003b \}, using continuous EEG-fMRI at 1.5T, described bilateral thalamic BOLD signal increase, and widespread cortical decrease in a patient with juvenile absence epilepsy (JAE). Aghakhani et al \{Aghakhani et al., 2004\} with continuous EEG-fMRI at 1.5T found thalamic haemodynamic signal change, predominantly activation, in 12 of 15 patients who had GSW during scanning, in addition to symmetrical cortical activation or deactivation associated with GSW. Archer et al \{Archer et al., 2003\} found posterior cingulate cortex (PCC) deactivation in association with GSW in four of five IGE patients studied with EEG-triggered fMRI at 3 T. Laufs et al. $\{$ Laufs et al., 2005\} noted thalamic activation and widespread cortical deactivation in a patient studied on two occasions, and emphasized the distribution of the cortical deactivation; being cerebral areas thought to be most active during the conscious resting state, the "default mode" hypotheses \{Raichle et al., 2001\}.

We report on a large consecutive series of patients with GSW, including patients with different subtypes of IGE as well as SGE. We hypothesized that population specific 
BOLD effects to GSW could be identified using EEG-fMRI and a random effects group analysis in a large group of patients

\section{Materials and Methods}

\section{Patients}

Forty six patients, 30 with IGE and 16 with SGE, and frequent GSW discharges on recent interictal EEG were recruited from the epilepsy clinics at the National Hospital for Neurology and Neurosurgery, London, the National Society for Epilepsy, Chalfont St Peter and St Thomas' Hospital, London. The study was approved by the National Hospital for Neurology and Neurosurgery and the Institute of Neurology Joint Research Ethics Committee. Patients gave written informed consent.

Patients were grouped according to the ILAE 1989 classification scheme \{Commission on Classification and Terminology of the International League Against Epilepsy, 1989\}. These were IGE and its sub classifications - juvenile myoclonic epilepsy (JME), juvenile absence epilepsy (JAE), childhood absence epilepsy (CAE), and epilepsy with generalized tonic clonic seizures only (IGE-GTCS) - and secondary generalized epilepsy (SGE). The latter group had one or more of the following: atypical absences, an abnormal background EEG, and irregular GSW. In one patient there was electrographic evidence of a unilateral frontal focus (patient 43, see table 1 and figures 1 and 3B). All patients had normal structural MRI.

\section{EEG acquisition}

Ten channels of EEG referenced to Pz and two channels of precordial ECG were recorded using in house recording equipment \{Allen et al., 1998\} \{Allen et al., 2000\}. Gold disk electrodes fitted with $10 \mathrm{kOhm}$ resistors, to reduce possible MR induced 
currents \{Lemieux et al., 1997\}, were applied to the scalp, at FP2/ Fp1, F8/ F7, T4/ T3, T6/ T7, O2/O1, Fz (ground) and Pz according to the international 10-20 system (for methods see) $\{$ Krakow et al., 2000 . An impedance of $<22 \mathrm{kOhm}$ across each electrode pair was typically achieved ( $20 \mathrm{kOhm}$ attributable to the resistors in each electrode). Algorithms for pulse and image artifact reduction, that calculate and subtract an averaged artifact waveform, were used to recover the underlying EEG, allowing the visualization of the EEG during the image acquisition (online) and subsequent review (off-line) \{Allen et al., 1998\} \{Allen et al., 2000\}.

\section{MRI acquisition}

A 1.5T GE Horizon echospeed MRI scanner (Milwaukee, Wisc.) was used to acquire 700 BOLD sensitive echo-planar images (EPI) images (TE/TR 40/3000, 21 x 5 $\mathrm{mm}$ interleaved axial slices (acquired parallel to the intercommissural line), FOV 24 x 24 cm, 64x64 matrix) over a 35 minute session with continuous, simultaneous EEG. An additional four images were acquired at the start of each session and discarded to allow for T1 equilibration effects. Foam padding or a vacuum head cushion was used to help secure the EEG leads, minimize motion, and improve patient comfort. Two successive 35 minute scan sessions at the same sitting were acquired in 13 patients, giving 59 sessions in 46 patients.

\section{Data Analyses}

The SPM2 software package (www.fil.ion.ucl.ac.uk/spm/) was used for all image pre-processing and voxel based statistical analyses. Images were slice-time corrected to the middle slice and spatially realigned to the first scan of the series, and then spatially 
normalized to the MNI template supplied by SPM. Finally images were spatially smoothed using an isotropic Gaussian kernel (10 $\mathrm{mm}$ full width at half maximum).

The artifact corrected EEG was reviewed off-line and the onset and offset of GSW epochs were identified relative to the fMRI time series. These were used to construct a boxcar model of the active (GSW) versus rest (background) EEG state. This model was convolved with the canonical haemodynamic response function (HRF), as supplied by SPM2 (peak at 6 seconds relative to inset, delay of undershoot 16 seconds and length of kernel 32 seconds), its time and dispersion derivatives, to form regressors testing for GSW-related BOLD changes. The temporal derivative (TD) and dispersion derivative (DD) were used to accommodate variations in the canonical HRF \{Handwerker et al., 2004\} \{Henson et al., 2002\}. Spatial realignment parameters and their first order expansion were included as effects of no interest to model the linear and non-linear effects of motion \{Friston et al., 1996\}. Data and design matrices were high pass filtered at 128 seconds cutoff. An auto regression (AR(1)) model was used to estimate the intrinsic autocorrelation of the data \{Friston et al., 2000\}. No global scaling or normalization was performed, to preclude introducing apparent deactivations in the analysis that would be artifactual.

An F-contrast was used to assess the variance at each voxel explained by GSW. The resulting SPMs were thresholded at $\mathrm{p}<0.05$ using the correction for multiple comparisons based on random field theory \{Friston et al., 1991\}. The contrast estimate pertaining to the canonical HRF \{Henson et al., 2002\} was used to ascertain the direction of the BOLD response at the global maxima and at the local maxima of frontal, posterior superior parietal, and posterior cingulate cortices. 
A random effects group analysis was performed on each of the groups IGE, and SGE using the following two stage procedure to infer the average pattern at a population level \{Friston et al., 1999\}. The contrast estimates pertaining to HRF, TD and DD, were taken to a second level random effects group analysis using a one-way analysis of variance. The inter-session variability in the number of EEG events requires particular consideration for group analyses due to the risk of unbalanced designs \{Friston et al., $2005\}$. We therefore selected patient sessions in which the number of GSW events fell within one order of magnitude of each other (see table 2, sessions marked *), giving 18 IGE cases and 10 SGE cases for the group analyses. An F contrast at the second (i.e. the between subject) level was used to test for the variance explained by GSW across the group. We had insufficient patient numbers \{Desmond and Glover, 2002\} with GSW during scanning for group analysis in JME and JAE.

\section{$\underline{\text { Results }}$}

\section{Clinical features}

See table 1 for the patients' clinical features. Good quality EEG was obtained following pulse and gradient artifact subtraction, allowing identification of epileptiform discharges (for examples see figure 1). In 16 sessions (10 patients, marked $\dagger$ in table 1) no GSW was seen; these sessions were not considered further. Three further patients were excluded due to correlation between head motion and GSW events (marked $\$$ in table 1). The rate of occurrence of GSW events in the remaining 40 sessions (in 33 patients) varied between 1 and 189 per 35-minute scan session (mean 28, median 11). 


\section{Single subject results}

Significant BOLD signal changes were seen in 25 patients, (29 sessions, $73 \%$ of sessions containing GSW): in thalamus (15 patients), frontal cortex (FC) (23 patients), posterior parietal cortex (PPC)(19 patients) and PCC / precuneus (20 patients), with one or typically more of these areas involved in each case (table 2). In addition, BOLD change was also seen, to a variable extent in some patients in basal ganglia, cerebellum, brainstem, the sagittal sinus, or all lobes.

Figure 2 shows examples of BOLD response patterns in 3 patients with the different IGE syndromes JME, IGE-GTCS, JAE, and figure 3 in one case with SGE, illustrating the similarities of the cortical pattern in these distinct syndromes. Negative cortical changes predominated, although positive, and biphasic changes, were also seen (table 2). BOLD decreases in FC were seen in 14 sessions (8 IGE, 6 SGE), increases in 10 (5 IGE, 5 SGE), and biphasic changes in 2 (IGE), PPC decreases were seen in 17 (11 IGE, 6 SGE), increases in 3 (1 IGE, 2 SGE), and biphasic changes in 1 (SGE), and PCC / precuneus decreases in 15 (8 IGE, 7 SGE), increases in 6 ( 3 IGE, 3 SGE) and biphasic changes in 1 . The signal change in the thalamus was positive in 9 sessions, biphasic in 4 and negative in 3 sessions. In patient 43, with SGE and EEG evidence of left frontal epileptogenicity a small area of frontal activation was seen in addition to a more widespread fronto-parietal cortical negative response (figure 3).

\section{Group results}

Table 3 gives the regions of significant BOLD changes revealed by the group analysis, with coordinates of local maxima and their respective $\mathrm{Z}$ score. This shows a group effect of thalamic signal increase in IGE, a biphasic change in SGE, and cortical 
signal decrease in a characteristic distribution of PPC, $\mathrm{PCC}$ / precuneus and FC in the IGE group and medial FC / anterior cingulate increase in the SGE group and biphasic thalamic change in the SGE group. Figure 4 shows the SPM $\{t\}$ of the HRF for the group analyses of the IGE, illustrating the cortical distribution of signal decrease and the thalamic activation.

\section{$\underline{\text { Discussion }}$}

BOLD signal changes were detected in $73 \%$ of sessions, a yield comparable to that previously reported \{Aghakhani et al., 2004\}. Those with no BOLD response had 7 events or less, all of which were of less than 2 seconds duration (table 2). The most striking feature of our results is the lack of changes in the primary cortices, except in a few cases, the primary visual cortex. Aghakhani et al (2004) similarly, found the cortical BOLD changes predominantly in a frontal and posterior distribution, similar to those seen here. They highlighted the symmetrical nature of their findings as well as the predominance of negative changes in cortex as seen here. We used a second level random effects group analysis to look for population specific BOLD effects to GSW. Sub threshold signal changes that are not seen at the individual level will become significant if they are present across subjects. Similarly if there is a high degree of variance at a particular voxel then activations that are seen on the individual level will not reach significance at the group level. We chose to show the results for both the individual analyses and the random effects group analysis to demonstrate 1) the degree of intersubject similarity, in different syndromes and variability within the same syndrome, at the individual level, that, at this present time preclude the use of EEG-fMRI as a 
clinical tool, and 2) population specific effects that allow inferences to be made about neuronal activity during GSW.

The selection of patients for this study was necessarily biased to those at the severe end of the spectrum often referred for optimization of medical treatment. Rarely were patients on no medication. Carbemazepine (CBZ) and gabapentin (GBP) are known to increase the amount of GSW in patients with IGE \{Kochen 2002\}, however they used if the initial diagnosis is incorrect, or in some improvements in generalized seizures are seen despite the relative contraindication. It is notable that 7 of 34 of our IGE patients with frequent discharges were taking $\mathrm{CBZ}$ or GBP at the time of the study. In the other EEG-fMRI studies 4 of 15 were taking CBZ in (Aghakhani et al. 2004) and 4 of 5 in (Archer et al.2003). The effect of anti epileptic medication on the neurovascular response is not known, although any effect is lessened by the fact that comparisons made here are within sessions. Even with optimal patient selection, the unpredictability and in cases paucity of GSW during scan sessions remains a limitation of EEG-fMRI. Activating procedures such as hyperventilation could be employed but are likely to introduce confounds due to variable effects on cerebral circulation and the BOLD response \{Kemna and Posse, 2001\}, whilst photic stimulation, sleep deprivation or drug withdrawal would run the risk of provoking generalized tonic clonic seizures.

The predominant finding in individual subject and group analyses was of thalamic activation and cortical BOLD negative response, consistent with recent EEG-fMRI reports $\{$ Salek-Haddadi et al., 2003b\} A Archer et al., 2003\} A Aghakhani et al., 2004\} \{Laufs et al., 2005\}. Thalamic signal change was seen in less than half of patients with IGE and almost all patients with SGE. This may be due to the greater occurrence of GSW 
in SGE cases compared to IGE (median SGE 44.5 versus IGE 8), and tended to be seen in those individual IGE cases with a higher number or longer duration of GSW. Primary cortical areas were spared, with changes occurring in frontal, parietal and temporal association areas. At the single subject level similar BOLD responses were seen across IGE syndromes and in SGE, suggesting that the predominant BOLD findings represent generic changes associated with GSW per se rather than syndrome specific patterns. This is unlikely due to syndrome misclassification \{Berkovic et al., 1987\} given the similarity of our findings in cases with very clear syndromic differences (figures 2 and 3).

It is not possible to infer causality of the BOLD changes relative to our modeled covariate, GSW; they may represent areas generating GSW, or alternatively reflect areas secondarily affected by GSW. This difficulty in inferring causality is in contrast to paradigm driven fMRI where it is generally safe to assume a primary association between the task or stimulus and the observed fMRI changes; in addition a prior hypothesis about a relatively well defined location of expected neural activity usually exits. In GSW however we have little prior anatomical hypothosis regarding the spatial extent of BOLD changes. We propose that thalamic activation seen here represents subcortical activity necessary for the maintenance of GSW \{Avoli et al., 2001\}. The absence of thalamic activation in a number of our cases may reflect low sensitivity of our model at $1.5 \mathrm{~T}$ $\{$ Laufs et al., 2005\}. The left frontal cortical activation in case 43 (figure 3B) requires further validation but likely represent an area of initiation of GSW, given its concordance with the left frontal EEG onset of GSW (figure 1d).

The cortical distribution of signal change, FC, PPC and PCC / precuneus, comprises areas of association cortex that are hypothesized, at rest, to be involved in an 
organized, baseline level of activity, a "default mode" of brain function \{Raichle et al., $2001\}$ \{Mazoyer et al., 2001\}. The default mode concept came from observations of consistent deactivations in meta analyses of different task related paradigms in fMRI, in addition to independent PET measurements of increased blood flow to these areas during awake conscious rest $\{$ Raichle et al., 2001\}. Activity in these areas as measured by PET is also altered during sleep, coma and anesthesia \{Laureys et al., 2004\}. Default mode areas likely represent part of a neural network subserving human awareness \{Laureys et al., 2004\}. Our study, and others \{Archer et al., 2003\} \{Aghakhani et al., 2004\} \{Laufs et al., 2005\}, shows alteration of activity in these regions during GSW, which would be consistent with the clinical manifestation of absence seizures. These findings are also consistent with PET findings of opioid release in association neocortex, most marked in parietal cortex and posterior cingulate, following absence seizures \{Bartenstein et al., $1993\}$.

The majority of cortical BOLD responses were negative (table 2), in keeping with other fMRI \{Salek-Haddadi et al., 2003b \} A Aghakhani et al., 2004\} \{Laufs et al., 2005\} as well as transcranial Doppler (TCD) \{ Sanada et al., 1988,Klingelhofer et al., 1991,Bode, 1992,Nehlig et al., 1996,De Simone et al., 1998,Diehl et al., 1998 3 and near infrared spectroscopy studies $\{$ Buchheim et al., 2004\} \{Haginoya et al., 2002\}. A number of lines of evidence suggest that BOLD negative responses represent a reduction in neural activity. In visual cortex, a reduction of BOLD signal, elicited by stimulating part of the visual field is due, primarily, to a reduction of neuronal activity $\{$ Shmuel et al., 2002\}. A decrease in BOLD signal is seen in the occipital cortex during auditory induced saccades $\{$ Wenzel et al., 2000\}, a manipulation known to suppress neuronal activity 
\{Duffy and Burchfiel, 1975\}. BOLD decreases have also been observed in the context of neuronal synchronization, modulated by sub-cortical structures $\{$ Parkes et al., 2004\}. Visual stimulation during sleep leads to occipital BOLD negative responses, confirmed as a decrease in $\mathrm{rCBF}$ with $\mathrm{H}_{2}{ }^{15} \mathrm{O}$ PET $\{$ Born et al., 2002\}. Similarly auditory BOLD negative responses occur on auditory stimulation during sleep, the amplitude and extent of which correlate positively with measures of EEG synchronization in sleep \{Czisch et al., 2004\}.

Although the majority of our cases showed cortical negative response a number of cases showed activation or biphasic time courses in the same areas. These did not differ significantly (2-sample t-tests, all $\mathrm{p}>0.8)$ from those with negative response in terms of the frequency of GSW, the number of events, their median duration or total duration during the scan session. Nevertheless activations were seen in a greater proportion of SGE than IGE patients. This would explain the absence of widespread cortical change in the SGE group analysis, where positive signal changes in some subjects and negative in others in the same region lead to a loss of this effect at the group level.

Our cases of cortical activation are compatible with the findings of others; cortical increases were seen in some cases with fMRI \{Aghakhani et al., 2004\} and in TCD an initial increase was seen prior to the more prolonged decrease \{Diehl et al., 1998\} \{De Simone et al., 1998\}. Similarly, SPECT and PET showed increases as well as decreases in cortical activity to spike wave \{Engel, Jr. et al., 1985\} \{Theodore et al., $1985\}$ \{Ochs et al., 1987\} \{Prevett et al., 1995\} \{Yeni et al., 2000\}. The terms "activation" and "deactivation" are operational terms that reflect measured vascular or metabolic responses from which inferences about neuronal activity are made, we have 
preferred to use the term negative BOLD response rather than deactivation as the neural correlates of these responses aren't yet clear. The terms have their origins in the field of PET \{Friston et al., 1990\}, in which measures are quantitative and more direct. In fMRI the modeling of signal changes is relative, i.e. a comparison between two states \{Bandettini et al., 1992\}. Given that 1) we, and others \{Aghakhani et al., 2004,SalekHaddadi et al., 2003 \} model GSW against an implicit baseline, i.e. active (GSW) against rest (background resting state activity), and 2) we detect changes in areas of association cortex, then the level of arousal or sleep reflecting resting state activity in these regions may affect the direction of detected BOLD signal changes. A negative response is therefore seen where there is a decrease or interruption of awake resting state activity; if however the patient is drowsy or asleep activity in these areas may be lower than that engendered by GSW thus giving rise to activations. Future studies of GSW with EEGfMRI need to take this into account, by modeling the background EEG in more detail or presenting the subject with a low grade task designed to maintain and monitor a constant level of vigilance.

\section{Conclusion}

In conclusion, we have shown that the main BOLD fMRI correlates of GSW consist of widespread cortical negative response in areas associated with normal brain activity during conscious rest. These likely reflect a decrease in neural activity, as a result of either synchronization or inhibition of cortical activity due to thalamo-cortical interactions. This is in keeping with the clinical features of absence seizures, i.e. a brief alteration of consciousness with minimal somatosensory or motor manifestations. 
EEG-fMRI of GSW

Hamandi K 


\section{Acknowledgments}

We thank Dr Michael Koutroumanidis for referring patients, Mr Phillip Allen and Mr Nathan Thoms for technical support with the EEG system and Dr Mark Symms, Dr

Philip Boulby and Professor Gareth Barker for fMRI sequence programming, acquisition and image reconstruction. This work was funded by the Wellcome Trust, grant number 067176 (KH, LL), and Medical Research Council, grant number 69812763 (ASH) and the Deutsche Forschungsgemeinschaft, grant number LA 1452/3-1)(HL). The National Society for Epilepsy (NSE) supports the NSE MRI unit. 


\section{$\underline{\text { References }}$}

Aghakhani Y, Bagshaw A P, Benar C G, Hawco C, Andermann F, Dubeau F, Gotman J. fMRI activation during spike and wave discharges in idiopathic generalized epilepsy. Brain 2004; (127): 1127-1144.

Al Asmi A, Benar C G, Gross D W, Khani Y A, Andermann F, Pike B, Dubeau F, Gotman J. fMRI activation in continuous and spike-triggered EEG-fMRI studies of epileptic spikes. Epilepsia 2003; (44): 1328-1339.

Allen P J, Josephs O, Turner R. A Method for Removing Imaging Artifact from Continuous EEG Recorded during Functional MRI. NeuroImage 2000; (12): 230-239. Allen P J, Polizzi G, Krakow K, Fish D R, Lemieux L. Identification of EEG Events in the MR Scanner: The Problem of Pulse Artifact and a Method for Its Subtraction. NeuroImage 1998; (8): 229-239.

Archer J S, Abbott D F, Waites A B, Jackson G D. fMRI "deactivation" of the posterior cingulate during generalized spike and wave. NeuroImage 2003; (20): 1915-1922. Avoli M, Rogawski M A, Avanzini G. Generalized epileptic disorders: an update. Epilepsia 2001; (42): 445-457.

Bandettini P A, Wong E C, Hinks R S, Tikofsky R S, Hyde J S. Time course EPI of human brain function during task activation. Magn Reson Med 1992; (25): 390-397. Bartenstein P A, Duncan J S, Prevett M C, Cunningham V J, Fish D R, Jones A K, Luthra S K, Sawle G V, Brooks D J. Investigation of the opioid system in absence seizures with positron emission tomography. J Neurol Neurosurg Psychiatry 1993; (56): 1295-1302. Berkovic S F, Andermann F, Andermann E, Gloor P. Concepts of absence epilepsies: discrete syndromes or biological continuum? Neurology 1987; (37): 993-1000. 
Bode H. Intracranial blood flow velocities during seizures and generalized epileptic discharges. Eur J Pediatr 1992; (151): 706-709.

Born A P, Law I, Lund T E, Rostrup E, Hanson L G, Wildschiodtz G, Lou H C, Paulson

O B. Cortical deactivation induced by visual stimulation in human slow-wave sleep.

NeuroImage 2002; (17): 1325-1335.

Buchheim K, Obrig H, Pannwitz W, Muller A, Heekeren H, Villringer A, Meierkord H.

Decrease in haemoglobin oxygenation during absence seizures in adult humans. Neurosci Lett 2004; (354): 119-122.

Commission on Classification and Terminology of the International League Against Epilepsy. Proposal for revised classification of epilepsies and epileptic syndromes. Commission on Classification and Terminology of the International League Against Epilepsy. Epilepsia 1989; (30): 389-399.

Czisch M, Wehrle R, Kaufmann C, Wetter T C, Holsboer F, Pollmacher T, Auer D P. Functional MRI during sleep: BOLD signal decreases and their electrophysiological correlates. Eur J Neurosci 2004; (20): 566-574.

De Simone R, Silvestrini M, Marciani M G, Curatolo P. Changes in cerebral blood flow velocities during childhood absence seizures. Pediatr Neurol 1998; (18): 132-135.

Desmond J E, Glover G H. Estimating sample size in functional MRI (fMRI) neuroimaging studies: statistical power analyses. J Neurosci Methods 2002; (118): 115128.

Diehl B, Knecht S, Deppe M, Young C, Stodieck S R. Cerebral hemodynamic response to generalized spike-wave discharges. Epilepsia 1998; (39): 1284-1289. 
Duffy F H, Burchfiel J L. Eye movement-related inhibition of primate visual neurons. Brain Res 1975; (89): 121-132.

Duncan J S. Idiopathic generalized epilepsies with typical absences. J Neurol 1997; (244): 403-411.

Engel J, Jr., Lubens P, Kuhl D E, Phelps M E. Local cerebral metabolic rate for glucose during petit mal absences. Ann Neurol 1985; (17): 121-128.

Friston K J, Frith C D, Liddle P F, Dolan R J, Lammertsma A A, Frackowiak R S. The relationship between global and local changes in PET scans. J Cereb Blood Flow Metab 1990; (10): 458-466.

Friston K J, Frith C D, Liddle P F, Frackowiak R S. Comparing functional (PET) images: the assessment of significant change. J Cereb Blood Flow Metab 1991; (11): 690-699. Friston K J, Holmes A P, Price C J, Buchel C, Worsley K J. Multisubject fMRI studies and conjunction analyses. NeuroImage 1999; (10): 385-396.

Friston K J, Josephs O, Zarahn E, Holmes A P, Rouquette S, Poline J. To smooth or not to smooth? Bias and efficiency in fMRI time-series analysis. NeuroImage 2000; (12): 196-208.

Friston K J, Stephan K E, Lund T E, Morcom A, Kiebel S. Mixed-effects and fMRI studies. NeuroImage 2005; (24): 244-252.

Friston K J, Williams S, Howard R, Frackowiak R S, Turner R. Movement-related effects in fMRI time-series. Magn Reson Med 1996; (35): 346-355.

Gloor P. Generalized cortico-reticular epilepsies. Some considerations on the pathophysiology of generalized bilaterally synchronous spike and wave discharge. Epilepsia 1968; (9): 249-263. 
Haginoya K, Munakata M, Kato R, Yokoyama H, Ishizuka M, Iinuma K. Ictal cerebral haemodynamics of childhood epilepsy measured with near-infrared spectrophotometry. Brain 2002; (125): 1960-1971.

Handwerker D A, Ollinger J M, D'Esposito M. Variation of BOLD hemodynamic responses across subjects and brain regions and their effects on statistical analyses. Neuroimage 2004; (21): 1639-1651.

Henson R N, Price C J, Rugg M D, Turner R, Friston K J. Detecting latency differences in event-related BOLD responses: application to words versus nonwords and initial versus repeated face presentations. NeuroImage 2002; (15): 83-97.

Holmes G L, McKeever M, Adamson M. Absence seizures in children: clinical and electroencephalographic features. Ann Neurol 1987; (21): 268-273.

Jasper H, Drooglever-Fortuyn J. Experimental studies on the functional anatomy of petit mal epilepsy. Res Publ Assoc Nerv Ment Dis 1947; (26): 272-298.

Kemna L J, Posse S. Effect of respiratory $\mathrm{CO}(2)$ changes on the temporal dynamics of the hemodynamic response in functional MR imaging. NeuroImage 2001; (14): 642-649. Klingelhofer J, Bischoff C, Sander D, Wittich I, Conrad B. Do brief bursts of spike and wave activity cause a cerebral hyper- or hypoperfusion in man? Neurosci Lett 1991; (127): 77-81.

Krakow K, Allen P J, Lemieux L, Symms M R, Fish D R. Methodology: EEG-correlated fMRI. Adv Neurol 2000; (83): 187-201.

Krakow K, Woermann F G, Symms M R, Allen P J, Lemieux L, Barker G J, Duncan J S, Fish D R. EEG-triggered functional MRI of interictal epileptiform activity in patients with partial seizures. Brain 1999; (122 ( Pt 9)): 1679-1688. 
Laufs H, Lengler U, Hamandi K, Kleinschmidt A, Krakow K. Linking generalized spike and wave discharges and resting state brain activity using EEG/fMRI in a patient with absence seizures. Epilepsia. in press. 2005.

Laureys S, Owen A M, Schiff N D. Brain function in coma, vegetative state, and related disorders. Lancet Neurology 2004; (3): 537-546.

Lemieux L, Allen P J, Franconi F, Symms M R, Fish D R. Recording of EEG during fMRI experiments: patient safety. Magnetic Resonance in Medicine: Official Journal of the Society of Magnetic Resonance in Medicine / Society of Magnetic Resonance in Medicine 1997; (38): 943-952.

Lemieux L, Salek-Haddadi A, Josephs O, Allen P, Toms N, Scott C, Krakow K, Turner R, Fish D R. Event-related fMRI with simultaneous and continuous EEG: description of the method and initial case report. Neuroimage 2001; (14): 780-787.

Marcus E M, Watson C W. Symmetrical epileptogenic foci in monkey cerebral cortex. Mechanisms of interaction and regional variations in capacity for synchronous discharges. Arch Neurol 1968; (19): 99-116.

Mazoyer B, Zago L, Mellet E, Bricogne S, Etard O, Houde O, Crivello F, Joliot M, Petit L, Tzourio-Mazoyer N. Cortical networks for working memory and executive functions sustain the conscious resting state in man. Brain Res Bull 2001; (54): 287-298.

Nehlig A, Vergnes M, Waydelich R, Hirsch E, Charbonne R, Marescaux C, Seylaz J. Absence seizures induce a decrease in cerebral blood flow: human and animal data. $\mathbf{J}$ Cereb Blood Flow Metab 1996; (16): 147-155.

Niedermeyer E, Laws E R, Jr., Walker E A. Depth EEG findings in epileptics with generalized spike-wave complexes. Arch Neurol 1969; (21): 51-58. 
Ochs R F, Gloor P, Tyler J L, Wolfson T, Worsley K, Andermann F, Diksic M, Meyer E, Evans A. Effect of generalized spike-and-wave discharge on glucose metabolism measured by positron emission tomography. Ann Neurol 1987; (21): 458-464.

Ogawa S, Lee T M, Kay A R, Tank D W. Brain magnetic resonance imaging with contrast dependent on blood oxygenation. Proc Natl Acad Sci 1990; (87): 9868-9872. Parkes L M, Fries P, Kerskens C M, Norris D G. Reduced BOLD response to periodic visual stimulation. NeuroImage 2004; (21): 236-243.

Prevett M C, Duncan J S, Jones T, Fish D R, Brooks D J. Demonstration of thalamic activation during typical absence seizures using H2(15)O and PET. Neurology 1995; (45): 1396-1402.

Raichle M E, MacLeod A M, Snyder A Z, Powers W J, Gusnard D A, Shulman G L. A default mode of brain function. Proc Natl Acad Sci 2001; (98): 676-682.

Salek-Haddadi A, Friston K J, Lemieux L, Fish D R. Studying spontaneous EEG activity with fMRI. Brain Res Rev 2003a; (43): 110-133.

Salek-Haddadi A, Lemieux L, Merschhemke M, Friston K J, Duncan J S, Fish D R. Functional magnetic resonance imaging of human absence seizures. Ann Neurol 2003b; (53): 663-667.

Sanada S, Murakami N, Ohtahara S. Changes in blood flow of the middle cerebral artery during absence seizures. Pediatr Neurol 1988; (4): 158-161.

Shmuel A, Yacoub E, Pfeuffer J, Van de Moortele P F, Adriany G, Hu X, Ugurbil K. Sustained negative BOLD, blood flow and oxygen consumption response and its coupling to the positive response in the human brain. Neuron 2002; (36): 1195-1210. 
Theodore W H, Brooks R, Margolin R, Patronas N, Sato S, Porter R J, Mansi L,

Bairamian D, DiChiro G. Positron emission tomography in generalized seizures.

Neurology 1985; (35): 684-690.

Timofeev I, Steriade M. Neocortical seizures: initiation, development and cessation.

Neuroscience 2004; (123): 299-336.

Velasco M, Velasco F, Velasco A L, Lujan M, Vazquez d M. Epileptiform EEG activities of the centromedian thalamic nuclei in patients with intractable partial motor, complex partial, and generalized seizures. Epilepsia 1989; (30): 295-306.

Wenzel R, Wobst P, Heekeren H H, Kwong K K, Brandt S A, Kohl M, Obrig H, Dirnagl

U, Villringer A. Saccadic suppression induces focal hypooxygenation in the occipital cortex. J Cereb Blood Flow Metab 2000; (20): 1103-1110.

Williams D. A study of thalamic and cortical rhythms in petit mal. Brain 1953; (76): 50-

69.

Yeni S N, Kabasakal L, Yalcinkaya C, Nisli C, Dervent A. Ictal and interictal SPECT findings in childhood absence epilepsy. Seizure 2000; (9): 265-269. 
$\underline{\text { Table } 1}$

\begin{tabular}{|c|c|c|c|c|}
\hline ID no & $\begin{array}{l}\text { Age } \\
(\mathbf{y r s})\end{array}$ & Seizure type frequency (age onset/yrs) & Antiepileptic Drugs & $\begin{array}{l}\text { Frequency of } \\
\text { GSW (Hz) }\end{array}$ \\
\hline \multicolumn{5}{|l|}{ JAE } \\
\hline 1 & 19 & GTCS 5/yr (15) & LEV, LTG & $2.5-3$ \\
\hline $2^{*}$ & 24 & GTCS 4/yr (13) & CBZ, ESM, LTG, TPM & 3 \\
\hline $3^{*}$ & 18 & GTCS 2/mth (14) & LTG & $3-4$ \\
\hline 4 & 43 & GTCS 5/yr (9) & CBZ, LTG & 3 \\
\hline $5^{*}$ & 43 & GTCS 4 /yr (13) & CLB, LTG, VPA & 3 \\
\hline $6^{*}$ & 22 & Abs 4-5 wk (9) $\quad$ GTCS 6/yr (16) & LTG, VPA & 4 \\
\hline 7 & 22 & Abs 2-3 day (8) $\quad$ GTCS 2/mth (19) & ESM, LTG, TPM & $3-4$ \\
\hline 8 & 54 & Abs 1/mth (19) GTCS none for 10yrs (19) & CBZ, PHT, PB, VPA & $3-4$ \\
\hline 9* & 18 & Abs weekly (15) & Nil & $2.5-3$ \\
\hline 10 & 19 & GTCS $<1$ yr (7) & CBZ, VPA & $2.5-3$ \\
\hline 11 & 33 & Abs daily (teens) & LTG, VPA & 3 \\
\hline $12 \dagger$ & 33 & Abs 10/day (10) $\quad$ GTCS 5/yr (14) & LTG, VPA & 3 \\
\hline $13 \dagger$ & 37 & Abs 1/mth (4) $\quad$ GTCS 2/yr (11) & LTG, OXC, PB, VPA & 3 \\
\hline $14 * \dagger$ & 36 & Abs weekly (3) GTCS 3 in total (6) & LTG & 3 \\
\hline \multicolumn{5}{|c|}{ Age range: $18-54$ yrs, mean 30, median 28.5. sex ratio M:F - 4:3. } \\
\hline \multicolumn{5}{|l|}{ JME } \\
\hline 15 & 55 & Abs resolved (13) GTCS 1-2/yr (13) $\quad$ MJ 2-3/wk (teens) & CLB, LEV, LTG & 3 \\
\hline $16 \ddagger$ & 20 & Abs daily (13) $\quad$ GTCS 2/mth (13) $\quad$ MJ daily (18), & CLN, TPM, VPA & $2-3$ \\
\hline $17^{*}$ & 59 & GTCS 1/wk (5) $\quad$ MJ several/wk(10) & CBZ, PB, VPA & 4 \\
\hline 18 & 20 & Abs 3/d (<10) $\quad$ GTCS 1/mth (13) $\quad$ MJ resolved (14) & LEV & 3 \\
\hline 19 & 41 & GTCS 1/mth (15) $\quad$ MJ resolved (15) & CLB, LTG, PHT, VPA & 3 \\
\hline $20^{*}$ & 18 & Abs 2-3/wk (15) GTCS<1/yr(16) MJ daily(15) & LEV, LMT, VPA & $4-5$ \\
\hline $21^{*}$ & 37 & Abs $10 \mathrm{~d}(7) \quad$ GTCS $2 / \mathrm{yr}(12)$ & CLB, GBP, VPA & $2-3$ \\
\hline 22 & 18 & MJ resolved (14) & VPA & 3 \\
\hline $23 * \dagger$ & 20 & Abs 3 wk (19), $\quad$ GTCS 1/yr (18) & CLN, LTG & $2-3$ \\
\hline \multicolumn{5}{|c|}{ Age range $18-59$ yrs, mean 34, median $33 . M: F-5: 4$} \\
\hline \multicolumn{5}{|l|}{ IGE-GTCS } \\
\hline 24 & 33 & GTCS free 2 yrs (11) & LTG & 4 \\
\hline $25^{*}$ & 34 & GTCS 3 in total (12) & CBZ & 3 \\
\hline 26 & 32 & GTCS 4 in total (22) & CBZ, CLN & $3-4$ \\
\hline $27 \dagger$ & 21 & GTCS $1 /$ mth (1) & CLB, LTG, VPA & $2.5-3$ \\
\hline $28 \dagger$ & 48 & GTCS $1<10$ yrs (7) & VPA & $2-3$ \\
\hline $29 * \dagger$ & 24 & GTCS 1 in total & Nil & $2-3$ \\
\hline \multicolumn{5}{|c|}{ Age range $21-48$, mean 32, median 32. M:F 2:1 } \\
\hline \multicolumn{5}{|l|}{ CAE } \\
\hline 30 & 23 & GTCS $1 /$ mth (12) & LEV & 3 \\
\hline $31 \ddagger$ & 26 & Abs 15/d (8) & Nil & 3 \\
\hline $32 \ddagger$ & 53 & Abs several/day (10) & DZP & 4 \\
\hline
\end{tabular}


EEG-fMRI of GSW

Hamandi K

\begin{tabular}{|c|c|c|c|c|c|c|}
\hline SGE & & & & & & \\
\hline 33 & 29 & Abs daily (20) & GTCS < 1 yr (23) & & LTG, VPA & 2-3 abn bkgd \\
\hline 34 & 29 & Abs 3/d (16) & GTCS <1 yr (14) & & ACE, CBZ, VPA & $1.5-2$ \\
\hline 35 & 21 & Abs daily (10) & GTCS $<1$ yr $(10)$ & tonic sz 1/wk (10) & ACE, CBZ, CLN & $2-3$ \\
\hline 36 & 19 & Abs 6-8/ d ( 7) & GTCS 6/yr (7) & & LEV, LTG, TGB & 3 abn bkgd \\
\hline 37 & 22 & Abs 2/wk (8) & GTCS resolved (9) & MJ 2/wk & CLN, LTG, OXC, VPA & $2-3$ \\
\hline 38 & 26 & & GTCS 2-3/yr (14) & drop attacks $6 / \mathrm{yr}(26)$ & LTG, TPM & $2-3$ \\
\hline 39 & 26 & Abs 2-3/wk (9) & GTCS < 1/yr (12) & atonic sz 1/wk (10) & CLN, LTG, OXC & 2 abn bkgd \\
\hline 40 & 74 & Abs daily (teens) & GTCS 2/mth(14) & & PHT, TPM & $2.5-3$ \\
\hline 41 & 22 & & GTCS 6/mth (11) & & CBZ, TPM & 3 \\
\hline 42 & 38 & Abs 10-20/d (7) & GTCS 0-4/mth (11) & & CBZ, LEV & $2-3$ \\
\hline 43 & 21 & Abs 8/mth (13) & GTCS 4/yr (18) & & CBZ, LEV, VPA & $2 \quad \mathrm{~L}>\mathrm{R}, \max \mathrm{F} 3$ \\
\hline $44 \dagger$ & 40 & Abs 1-2/mth, (11) & GTCS 2/yr, (11) & & CBZ, PHT, TGB & 3 \\
\hline $45 \dagger$ & 19 & & GTCS 2/mth (2) & & LTG, TPM & $2-3$ abn bkgd \\
\hline $46 * \dagger$ & 47 & Abs 15/d (2) & GTCS 3/mth (4) & & PHT & $3-4$ \\
\hline
\end{tabular}

Table 1. Clinical details of patients studied based on ILAE diagnostic categories, showing seizure type and frequency, age at onset, medication at time of study and frequency of GSW. Structural MRI in all patients was normal. The sub classification of IGE is often a source of debate, with a degree of overlap between syndromes, and some would therefore argue they represent a continuum rather than specific disease entities. For example patient \#10, whilst the age of onset 7 years might suggest CAE, we prefer to classify as JAE on the basis of GTCS occurring with the onset of epilepsy, similarly patient \#32 whilst the age of onset of 10 might suggest JAE has never had a GTCS, this would be unusual for JAE, hence we include them in CAE \{Panaytopolous 2002\}. JAE, Juvenile absence epilepsy; JME, Juvenile Myoclonic Epilepsy; IGE-GTC, idiopathic generalized epilepsy with generalized tonic clonic seizures only; SGE. secondary generalized epilepsy; abs, absence seizures; MJ, myoclonic jerks; GTCS, generalized tonic clonic seizures; ACE, actetazolamide; CBZ, carbemazepine; CLB, clobazam; CLN, clonazepam; DZP, diazepam; ESM, ethosuxamide; GBP, gabapentin; 
LEV, leviteracetam; LTG, lamotrigine; OXC, oxcarbazapine; PB, phenobarbitone; PHT, phenytoin; TGB, tiagabine; TPM, topiramate; VPA, sodium valproate; M, male; F, female; abn, abnormal; bkgd, background; F3, left frontal electrode; d, day; wk, week; mth, month; yr, year; sz, seizure. ${ }^{*}$ patients studied in two successive sessions, $\dagger$ patients in whom no GSW was seen during scanning, $\$$ patients excluded due to correlated motion. 
EEG-fMRI of GSW

Hamandi K

$\underline{\text { Table }} \underline{2}$

\begin{tabular}{|c|c|c|c|c|c|c|c|c|}
\hline \multirow{2}{*}{ 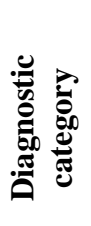 } & \multirow{2}{*}{$\begin{array}{c}\text { Id } \\
\text { No. }\end{array}$} & \multirow{2}{*}{$\begin{array}{c}\text { No. of } \\
\text { GSW } \\
\text { Events }\end{array}$} & \multirow{2}{*}{$\begin{array}{c}\text { Duration of } \\
\text { GSW events. } \\
\text { Median (range) } \\
\text { (seconds) }\end{array}$} & \multicolumn{5}{|c|}{ Regions of significant fMRI signal change } \\
\hline & & & & Thalamus & Frontal & Parietal & $\begin{array}{c}\text { Posterior } \\
\text { cingulate / precuneus }\end{array}$ & Other \\
\hline \multirow{15}{*}{$\sum_{5}^{5}$} & $1 *$ & 9 & $2.1(1.6-8.1)$ & - & $\uparrow \mathrm{B}(\mathrm{L} \mathbf{m})$ & $\downarrow \mathrm{B}$ & $\uparrow \mathrm{B}$ & $\uparrow \mathrm{ss}$, cereb, temp \\
\hline & $2 \mathrm{a}$ & 3 & $7.3(4.4-7.7)$ & - & $\uparrow \mathrm{B}(\mathrm{L} \mathrm{m})$ & $\downarrow \mathrm{B}$ & - & $\uparrow$ L temp \\
\hline & $2 \mathrm{~b}$ & 1 & 5.9 & - & $\uparrow \mathrm{R} \mathrm{m}$ & - & - & $\uparrow \mathrm{B}$ temp \\
\hline & $3 *$ & 7 & $1.3(0.9-3.3)$ & $\downarrow R$ & $\uparrow \mathrm{B}$ & - & - & $\uparrow \mathrm{B}$ head caudate $(\mathrm{R} \mathbf{~ m})$ \\
\hline & $4 *$ & 4 & $2.6(1.0-3.0)$ & - & - & - & - & - \\
\hline & $5 \mathrm{a}$ & 18 & $0.6(0.4-3.6)$ & $\uparrow \mathrm{B}$ & $\uparrow \mathrm{B}$ & - & $\uparrow \mathrm{B}$ & $\uparrow$ ss, cereb (R m), B occ \\
\hline & $5 b^{*}$ & 16 & $0.7(0.4-1.7)$ & - & - & - & $\uparrow \mathrm{B}$ & $\uparrow$ cereb $(\mathrm{L} \mathrm{m}), \mathrm{B}$ occ \\
\hline & $6 a$ & 4 & $1.9(1.3-3.0)$ & - & - & - & - & - \\
\hline & $6 b^{*}$ & 17 & $1.3(0.4-4.3)$ & - & - & - & - & $\downarrow$ L inf occ m \\
\hline & 7 & 2 & $4.3(3.4-5.3)$ & $\uparrow \mathrm{B}$ & $\uparrow \mathrm{B}(\mathrm{L} \mathrm{m})$ & $\uparrow \mathrm{R}$ & - & $\uparrow \mathrm{ss}$ \\
\hline & $8^{*}$ & 8 & $2.1(1.0-5.3)$ & - & $\downarrow \mathrm{B}(\mathrm{L} \mathbf{~ m})$ & $\downarrow \mathrm{B}$. & $\downarrow \mathrm{B}$ & - \\
\hline & $9 a^{*}$ & 8 & $1.9(0.7-3.6)$ & - & $\uparrow \mathrm{B}$ & $\downarrow \mathrm{B}$ & $\downarrow \mathrm{B}$ & $\uparrow \mathrm{ss} \mathbf{m}$ \\
\hline & $9 \mathrm{~b}$ & 2 & $0.6(0.6-0.7)$ & - & - & - & - & - \\
\hline & $10^{*}$ & 11 & $1.3(0.9-2.6)$ & - & - & - & - & - \\
\hline & 11 & 189 & $1.6(0.3-73.9)$ & $\uparrow \mathrm{B}$ & $\downarrow \mathrm{B}(\mathrm{L} \mathbf{~ m})$ & $\downarrow \mathrm{B}$ & $\downarrow \mathrm{B}$ & $\downarrow$ ss, cereb \\
\hline \multirow{9}{*}{$\sum^{\text {될 }}$} & $15^{*}$ & 17 & $0.7(0.4-5.3)$ & - & - & - & - & $\downarrow$ occ $\mathbf{m}, \uparrow$ R BS \\
\hline & $17 a^{*}$ & 7 & $1.4(1.1-1.6)$ & - & - & - & - & - \\
\hline & $17 \mathrm{~b}$ & 1 & 1.4 & - & - & - & - & - \\
\hline & $18^{*}$ & 25 & $1.3(0.4-3.4)$ & $\uparrow \mathrm{B}(\mathrm{L} \mathrm{m})$ & $\uparrow \mathrm{B}$ & - & $\uparrow \mathrm{B}$ & $\uparrow$ occ, B temp \\
\hline & $19 *$ & 4 & $0.6(0.6-0.9)$ & - & - & - & - & - \\
\hline & $20 *$ & 7 & $1.6(0.7-1.7)$ & - & - & - & - & - \\
\hline & $21 \mathrm{a}$ & 60 & $1.4(0.4-8.4)$ & $\downarrow \mathrm{B}(\mathrm{L} \mathbf{~ m})$ & $\downarrow \mathrm{B}$ & $\downarrow \mathrm{B}$ & $\downarrow \mathrm{B}$ & $\downarrow \mathrm{BS}$ \\
\hline & $21 b$ & 89 & $1.0(0.3-5.4)$ & $\uparrow \mathrm{B}$ & $\downarrow \mathrm{B}$ & $\downarrow R$ & $\downarrow \mathrm{B}$ & $\uparrow \mathrm{BS} \mathbf{m}$ \\
\hline & $22 *$ & 11 & $9(2.4-15.7)$ & - & $\downarrow \mathrm{B}$ & $\downarrow \mathrm{B}(\mathrm{L} \mathrm{m})$ & $\downarrow \mathrm{B}$ & - \\
\hline \multirow{4}{*}{ 我记 } & $24 *$ & 6 & $1.1(0.9-1.7)$ & - & - & - & - & - \\
\hline & $25 a$ & 33 & $1.6(0.6-3.9)$ & - & $\downarrow \mathrm{B}$ & $\downarrow \mathrm{B}(\mathrm{L} \mathbf{m})$ & $\downarrow \mathrm{B}$ & - \\
\hline & $25 b^{*}$ & 24 & $1.4(0.6-4.1)$ & - & $\downarrow \mathrm{B}$ & $\downarrow \mathrm{B}(\mathrm{L} \mathbf{m})$ & - & - \\
\hline & $26^{*}$ & 5 & $1.1(0.7-1.4)$ & - & - & - & - & - \\
\hline
\end{tabular}


EEG-fMRI of GSW

Hamandi K

\begin{tabular}{|c|c|c|c|c|c|c|c|c|}
\hline CAE & $32 *$ & 17 & 2.1 & $\uparrow \mathrm{B}$ & $\downarrow \mathrm{B}$ & $\downarrow \mathrm{B}$ & $\downarrow \mathrm{B}$ & $\uparrow$ cereb m \\
\hline \multirow{11}{*}{ 窇 } & $33^{*}$ & 25 & $1.1(0.3-2.0)$ & $\uparrow R$ & $\downarrow \mathrm{B}$ & $\downarrow \mathrm{B}(\mathrm{R} \mathbf{~ m})$ & $\downarrow \mathrm{B}$ & - \\
\hline & $34^{*}$ & 55 & $13.7(1.0-82.4)$ & $\uparrow \mathrm{B}$ & $\downarrow \mathrm{B}$ & $\uparrow \mathrm{B}$ & $\downarrow \mathrm{B}(\mathrm{R} \mathbf{~ m})$ & $\downarrow$ all lobes, cereb, BS, BG \\
\hline & $35^{*}$ & 32 & $10.9(1.3-82.4)$ & $\downarrow \mathrm{B}$ & $\downarrow \mathrm{B}(\mathrm{L} \mathrm{m})$ & $\downarrow \mathrm{B}$ & $\downarrow \mathrm{B}$ & $\downarrow$ ss, cereb., temp, BS \\
\hline & $36^{*}$ & 9 & $7.1(5.0-12.6)$ & - & $\uparrow \mathrm{B}(\mathrm{R} \mathbf{m})$ & $\downarrow \mathrm{B}$ & $\downarrow \mathrm{B}$ & $\uparrow \mathrm{ss}, \downarrow \mathrm{BS}$ \\
\hline & $37 *$ & 78 & $2.6(0.6-15.0)$ & $\uparrow \mathrm{B}$ & $\uparrow \mathrm{B}$ & - & $\uparrow \mathrm{B}(\mathrm{L} \mathrm{m})$ & $\uparrow$ occ \\
\hline & $38 *$ & 43 & $5.7(0.7-12.3)$ & $\uparrow \mathrm{B}(\mathrm{R} \mathbf{m})$ & $\uparrow \mathrm{B}$ & $\uparrow \mathrm{B}$ & $\uparrow \mathrm{B}$ & $\uparrow \mathrm{ss}$, cereb, $\uparrow \mathrm{BS}$ \\
\hline & $39 *$ & 68 & $6.6(0.4-39)$ & $\uparrow \mathrm{B}(\mathrm{R} \mathbf{m})$ & $\uparrow \mathrm{B}$ & $\uparrow \mathrm{B}$ & $\uparrow \mathrm{B}$ & $\uparrow$ cereb, temp, BS, BG \\
\hline & 40 & 3 & $6.9(1.9-8.6)$ & - & - & - & - & - \\
\hline & $41 *$ & 46 & $0.7(0.3-12.9)$ & $\uparrow \mathrm{B}$ & $\downarrow \mathrm{B}$ midline $(\mathrm{L} \mathbf{~ m})$ & $\downarrow \mathrm{B}$ & $\downarrow \mathrm{B}$ & $\downarrow$ cereb, $\uparrow$ BS, temp, occ \\
\hline & $42 *$ & 57 & $4.1(1.1-24.6)$ & - & $\downarrow \mathrm{B}$ & $\downarrow \mathrm{B}(\mathrm{R} \mathbf{~ m})$ & $\downarrow \mathrm{B}$ & $\downarrow$ temp \\
\hline & $43^{*}$ & 46 & $5.2(1.1-29.3)$ & $\uparrow \mathrm{L}$ & $\downarrow \mathrm{B}+$ area of $\mathrm{L} \uparrow$ & $\downarrow \mathrm{B}(\mathrm{R} \mathbf{~ m})$ & $\downarrow \mathrm{B}$ & $\downarrow \mathrm{BS}$ \\
\hline
\end{tabular}

Table 2. Summary of results for all sessions during which GSW activity occurred, detailing number and duration of

GSW epochs, and regions of significant BOLD signal change labelled in accordance with direction of HRF loading. All

SPMs corrected for multiple comparisons using random field theory $(\mathrm{p}<0.05)$.

Id no - identification number, corresponds to that in table 1. (a) and (b) are given for those studied with two sessions.

$\uparrow$ - increase, $\downarrow$ - decrease, $\uparrow$ - biphasic. B - bilateral, L - left, R - right, m - global maxima, BG - basal ganglia, BS -

brainstem, BG - basal ganglia, cereb - cerebellum, temp - temporal lobes, occ - occipital lobes, ss - sagittal sinus

(draining vein).

*indicates sessions included in second level group analysis. 
$\underline{\text { Table } 3}$

A. IGE group analysis

\begin{tabular}{cllll}
\hline \multicolumn{2}{c}{ Region } & $x, y, z$ & $\begin{array}{l}\text { Peak Z } \\
\text { score }\end{array}$ & $\begin{array}{l}\text { Weighting on } \\
\text { contrast estimate }\end{array}$ \\
\hline Parietal & & & \\
L & Angular Gyrus & $-44,-62,34$ & 5.96 & $\downarrow$ \\
R & Supramarginal Gyrus & $46,-51,32$ & 5.86 & $\downarrow$ \\
R & Posterior Cingulate & $6,-48,17$ & 5.23 & $\downarrow$ \\
Frontal & & & \\
L & Inferior Frontal Gyrus & $-51,16,16$ & 4.75 & $\downarrow$ \\
L & Inferior Frontal Gyrus & $-50,26,-15$ & 4.11 & $\downarrow$ \\
R & Inferior Frontal Gyrus & $53,35,7$ & 3.86 & $\downarrow$ \\
L & Inferior Frontal Gyrus & $-38,49,1$ & 3.82 & $\downarrow$ \\
R & Middle Frontal Gyrus & $53,23,23$ & 5.47 & $\downarrow$ \\
L & Superior Frontal Gyrus & $-12,61,23$ & 3.76 & $\downarrow$ \\
R & Superior Frontal Gyrus & $18,48,29$ & 3.59 & $\downarrow$ \\
Temporal & & & & \\
L & Fusiform Gyrus & $-51,-34,-20$ & 4.21 & $\downarrow$ \\
R & Middle Temporal Gyrus & $67,-28,-12$ & 4.51 & $\downarrow$ \\
L & Middle Temporal Gyrus & $-46,-52,3$ & 3.78 & $\downarrow$ \\
L & Parahippocampal Gyrus & $-16,-10,-13$ & 3.98 & $\downarrow$ \\
Subcortical & & & & \\
L & Caudate & $-14,10,5$ & 4.68 & $\downarrow$ \\
R & Caudate & $10,8,5$ & 4.13 & $\downarrow$ \\
R & Thalamus & $12,-11,4$ & 3.67 & $\uparrow$
\end{tabular}

B. SGE group analysis

\begin{tabular}{llll}
\hline Region & $x, y, x$ & $\begin{array}{l}\text { Peak Z } \\
\text { score }\end{array}$ & $\begin{array}{l}\text { Weighting on } \\
\text { contrast estimate }\end{array}$ \\
\hline Frontal & & & \\
L Cingulate Gyrus & $-8,10,42$ & 5.56 & $\uparrow$ \\
L Anterior Cingulate & $-8,33,2$ & 3.94 & $\uparrow$ \\
$\quad$ L Inferior Frontal Gyrus & $-50,5,27$ & 3.98 & $\uparrow$ \\
$\quad$ R Cingulate Gyrus & $10,9,33$ & 3.89 & $\uparrow$ \\
Subcortical & & & \\
$\quad$ L Thalamus & $-14,-15,4$ & 4.15 & $\uparrow$ \\
R Thalamus & $10,-15,3$ & 3.92 & $\uparrow$
\end{tabular}

Table 3. Brain regions that showed significant change on the group analysis. A: IGE cases and B: SGE cases. The weighting on the contrast is given to show the direction of BOLD signal change. L, left; R, right; hrf, canonical haemodynamic response; td, temporal derivative 


\section{Figure legends}

Fig 1. Examples of EEG recorded during scanning following pulse and image artifact subtraction, displayed as bipolar montage showing runs of generalized spike wave activity. a) patient \#1, JAE. b) patient \#25, IGE-GTCS. c) patient \#35, SGE d) patient \#43, SGE with evidence of L frontal epileptogenicity. ECG electrocardiogram, OSC scanner slice pulse used for EEG artifact correction and EEG-fMRI synchronization (7/second).

Fig 2. Examples of mean intensity projections from single subject SPM analyses showing cortical signal change with GSW involving symmetrical bi-frontal, biparietal, posterior cingulate/precuneus in three patients with different diagnostic syndromes a) patient \#10, JAE, b) patient \#22 JME c) patient \#25 IGE-GTCS. The fitted response for each global maximum (marked with red arrow on the SPM) is plotted on the right indicating the peristimulus time course and percent signal change. (SPMs corrected, $\mathrm{p}<0.05)$

Fig 3. Examples of mean intensity projections $\operatorname{SPM}\{\mathrm{F}\}$ from 2 patients with SGE whos EEGs are shown in figure 1. A color coded overlay of SPM $\{\mathrm{t}\}$ (redactivation and green deactivation) onto the surface render is shown for display purposes and a plot of the weighting on the contrast estimates also shown to indicate the direction of the signal change, both show a similar distribution on neagative BOLD to the IGE cases shown on figure 2, although to a greater extent due to the higher number of events during the scan session.. A) Patient \#35 shows widespread cortical deactivation sparing primary cortical areas. B) Patient \#43 is 
also shown as this illustrates in addition to the negative BOLD changes a small area of left frontal activation concordant with the EEG abnormality.

Fig 4. SPM $\{\mathrm{t}\}$ overlaid onto canonical brain of positive HRF (red) and negative HRF (green) (uncorrected $\mathrm{p}<0.001$ ) illustrating the thalamic and cortical distribution of BOLD changes to GSW in the IGE group analysis. This shows bilateral parietal [46 -51 32] and [-44 -62 34] and posterior cingulate / precuneus deactivation and [6 -48 17] thalamic activation [12 -11 4], the activation around the ventricles, we suspect is due to modeled changes in CSF pulsation as a result of the widespread haemodynamic changes occurring during GSW. 